\title{
Robotic-Assisted Simple Prostatectomy: A Systematic Review
}

\author{
Yakup Kordan 1,*®D, Abdullah Erdem Canda ${ }^{1}$, Ersin Köseoğlu ${ }^{1}$, Derya Balbay ${ }^{1}$, \\ M. Pilar Laguna ${ }^{2}$ and Jean de la Rosette ${ }^{2,3}$ \\ 1 Department of Urology, School of Medicine, Koc University, Zeytinburnu, 34010 Istanbul, Turkey; \\ ecanda@kuh.ku.edu.tr (A.E.C.); ekoseoglu@kuh.ku.edu.tr (E.K.); dbalbay@ku.edu.tr (D.B.) \\ 2 Department of Urology, School of Medicine, Istanbul Medipol University, 34083 Istanbul, Turkey; \\ m.p.lagunapes@gmail.com (M.P.L.); j.j.delarosette@gmail.com (J.d.l.R.) \\ 3 Amsterdam University Medical Centers, 1105 Amsterdam, The Netherlands \\ * Correspondence: ykordan@kuh.ku.edu.tr or kordanyakup@gmail.com; Tel.: +90-532-446-0737
}

Received: 5 May 2020; Accepted: 2 June 2020; Published: 9 June 2020

\begin{abstract}
Contemporary minimally invasive surgical (MIS) treatment options of patients with male Lower Urinary Tract Symptoms (LUTS) in men with prostate glands $>80 \mathrm{~mL}$ include Holmium Laser Enucleation Prostate (HoLEP), Thulium laser VapoEnucleation Prostate (ThuVEP), and Laparoscopic (LSP) or Robotic-Assisted Simple Prostatectomy (RASP). Implementing new laser technologies is costly, and the steep learning curve of these laser techniques limit their wide range use. This promoted the use of LSP and RASP in centers with readily established laparoscopy or robotic surgery programs. The aim of this study is to review case and comparative series of RASP. We systematically reviewed published data from 2008 to 2020 on RASP and have identified 26 non-comparative and 9 comparative case series. RASP has longer operation time but less time spent in hospital and less blood loss. The outcomes of improvements in symptom score, post-voiding residual urine (PVR), postoperative PSA decline, complications, and cost are similar when compared to open and laser enucleation techniques. These outcomes position RASP as a viable MIS treatment option for patients with male LUTS needing surgical treatment for enlarged prostates. Nevertheless, prospective, randomized controlled trials (RCTs) with multicenter and large sample size are needed to confirm the findings of this systematic review.
\end{abstract}

Keywords: benign prostate hyperplasia; miminally invasive simple prostatectomy; minimally invasive adenomectomy; robotic adenomectomy; robotic simple prostatectomy

\section{Introduction}

Male lower urinary tract symptoms (LUTS) caused by an enlarged prostate adversely affects many men and impairs their quality of life. Despite adequate medical therapy, surgical interventions are recommended in men with urinary retention, impaired renal function, and dilatation of upper urinary tract secondary to obstruction, recurrent urinary tract infections, recurrent hematuria, accompanying bladder stones, diverticulas, and refractory symptoms.

The "gold standard" surgical intervention for large prostate glands $>80$ gr has traditionally been open simple prostatectomy (OSP). Retropubic and suprapubic (transvesical) OSP are the most common and widespread techniques for large adenomas, especially in developing countries. However, OSP is usually associated with substantial perioperative complications and morbidity of up to $42 \%$, including prolonged catheterization time, increased estimated blood loss (EBL), length of hospital stay (LOS), and a transfusion rate of more than $24 \%$ [1]. Therefore, a variety of minimally invasive surgical techniques have emerged and extensively investigated for the treatment of large obstructing adenomas. 
The first laparoscopic (LSP) was reported in 2002 by Mariano et al. [2]. Since then, LSP was quickly adopted and widely used by urologists experienced in laparoscopy. In conjunction, published series of LSP increased every year until 2008 when the first report of robotic-assisted simple prostatectomy (RASP) was published in 2008 by Sotelo et al. [3].

The recent data on laser technologies such as holmium laser enucleation prostate (HoLEP) and thulium laser vapoenucleation prostate (ThuVEP) established these techniques as alternative approaches to OSP [4]. This has been adopted in two major guidelines recommendations. For large prostate glands $>80 \mathrm{~mL}$, American Urological Association (AUA) guidelines [5] recommend HoLEP, ThuVEP, or simple prostatectomy (SP) which can be performed in open, laparoscopic, or robotically according to the surgeon's expertise and discretion. On the other hand, the European Association of Urology (EAU) guidelines [6] recommend HoLEP, bipolar enucleation, and open simple prostatectomy (OSP) as first line treatment options. However, LSP and RASP, which are minimal invasive simple prostatectomy (MISP) techniques, are still regarded as feasible in men with prostate sizes $>80 \mathrm{~mL}$ needing surgical treatment in EAU guidelines. EAU guidelines also indicate the need of randomized controlled studies for further recommendation. An increasing body of evidence supports the role of HoLEP and ThuVEP in the treatment of BPH with large prostate $>80$ gr given they are "size independent" treatment approaches with favorable long-term outcomes $[4,7]$. However, some of these treatment options also enharbour several limitations. For example, to become sufficient to perform HoLEP, the medical procedure requires new equipment to perform such a surgery and has a steep learning curve. Several studies concluded that approximately 50 cases were needed for an efficient HoLEP surgery $[8,9]$. These limitations have led to the search for alternative new energies for enucleation and alternative surgical techniques. With the increasing use of robotic surgery in urology, several teams have explored the option to perform a robotic assisted simple prostatectomy (RASP) for large prostate glands. Robotic systems are widely distributed, and RASP is easy to implement and perform. RASP presents all the advantages of minimal invasiveness of LSP. Moreover, RASP provides three-dimensional vision, five degrees of movement, faster learning curve, especially regarding suture techniques, and comfortable operating position overcoming disadvantages of LSP. An increasing body of evidence confirms that RASP offers comparable outcomes to open and laser enucleation techniques for improvement in outcomes at a favorable low complication rate and costs [4]. Besides, RASP, compared with OSP, provides decreased length of hospital stay, blood loss, and transfusion prevalence [10].

Detailed scrutiny of the present literature revealed that there is no randomized controlled trial (RCT) which compares RASP with OSP or other treatment modalities. Recent systematic reviews and metanalyses compared MISP (RASP + LSP) with OSP or other endoscopic techniques such as lasers, vaporizations, and bipolar resections. However, there is not any recent systematic review or a metanalysis directly comparing RASP to other treatment modalities. This systematic review of RASP critically analyzes the current data available and provides recommendations for the use of RASP in clinical practice and possible implementation in guidelines.

\section{Methods}

\subsection{Evidence Acquisition}

We performed a structured, comprehensive review of the current literature for RASP. Eligible articles were identified through the electronic databases PUBMED, MEDLINE, and Web of Science. PRISMA guidelines were followed to select relevant papers. Figure 1 shows the article selection process according to PRISMA guidelines. The search employed the term "Benign prostatic hyperplasia" AND "minimally invasive simple prostatectomy" OR "robotic simple prostatectomy" OR "robotic adenomectomy". In addition, references in the reference sections of the identified publications were also added to the list. The raw data for this systematic review is publicly available through the Open Science Framework [11]. 

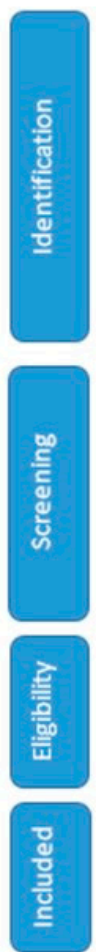

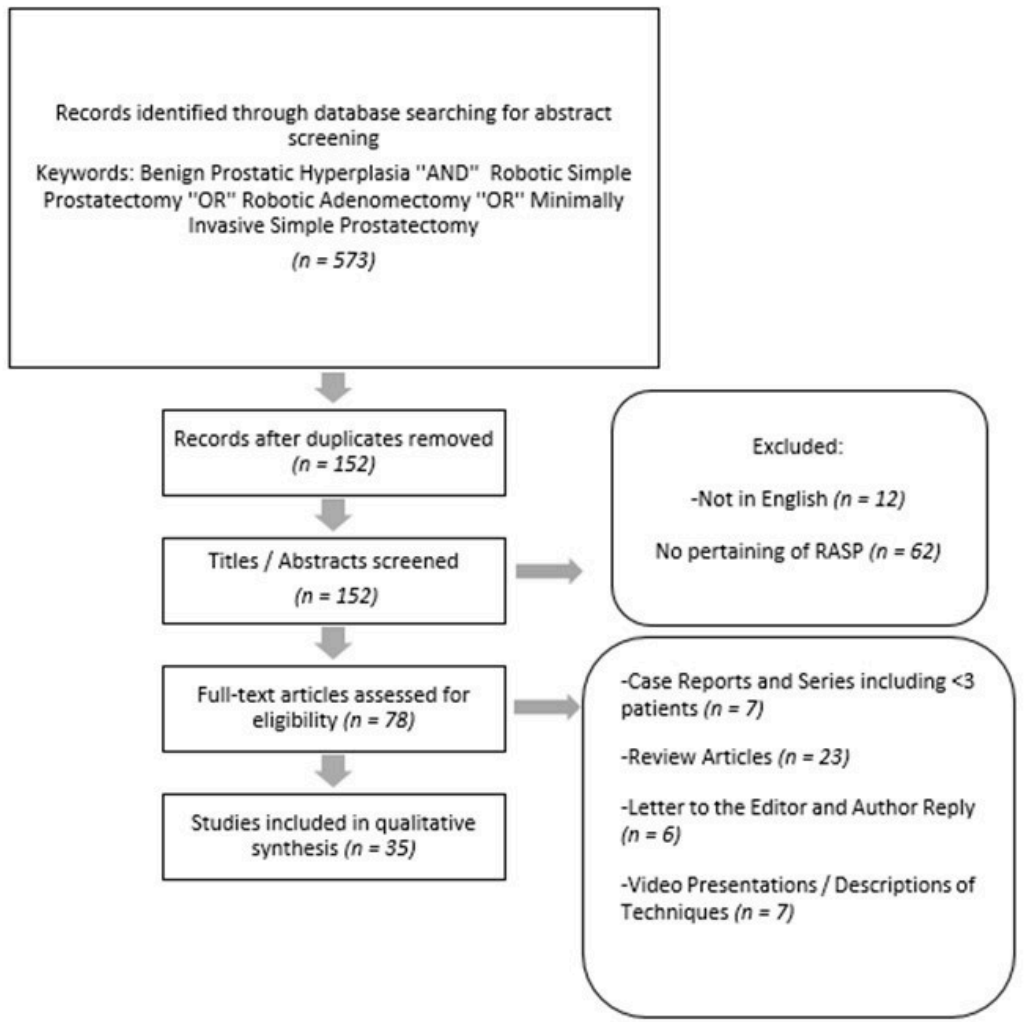

Figure 1. PRISMA 2009 flowchart for the article selection process.

\subsection{Inclusion and Exclusion Criteria}

Original articles reporting outcomes of case series and comparative studies of RASP, other minimally invasive techniques (HoLEP, Green Light laser enucleation, ThuVEP etc.), or OSP were eligible. Review articles, case reports, and case series with less than three patients were excluded. Search was not limited by region or language. However, articles published in English between 2008 (the first published RASP article) and February 2020 were included in this analysis. Each article's title, abstract and (when necessary) full articles were reviewed for their appropriateness and their relevance with regards to RASP by two authors (Y.K. and A.E.C.) and a third author (D.B.) resolved eventual discrepancies. Twenty-six original non-comparative and nine comparative case series were finally included in the evidence synthesis.

\subsection{Data Extraction and Outcome Measures}

Data variables of interest were age, surgical approach and technique, preoperative and postoperative maximum flow rates (Qmax), preoperative and postoperative International Prostate Symptom Score (IPSS), operative time (OT), estimated blood loss (EBL), postoperative day of catheter removal, length of hospital stay (LOS), complications, incontinence and erectile dysfunction (International Index of erectile function (IIEF) and Sexual Health Inventory for men (SHIM)). Complications like blood transfusion, urinary tract infection, urinary retention, and conversion to OSP were carefully analyzed. Clavien-Dindo classification of complications were also included. 


\section{Results}

We identified 573 relevant articles at our initial search. After omitting duplicates, articles not pertaining to RASP, and articles in languages other than English, 78 articles remained. 43 articles which include case reports, case series with $<3$ patients, reviews and meta-analyses, letters to editor, video presentations, and descriptions of techniques were removed from the study. Thirty-five studies were found to be eligible (26 case and 9 case comparative studies) for final analysis (Figure 1).

All 26 case studies $[3,10,12-35]$ included in the final analysis were non comparative case series with level 3 evidence. In nine comparative studies [36-44], outcomes of RASP were compared to outcomes of other minimally invasive modalities (OSP, HoLEP, ThuVEP) for the treatment of benign prostate hyperplasia (BPH). Unfortunately, there have been no randomized clinical trials among these studies conducted to date. When the outcomes of RASP patients from these comparative studies are added to case studies the total number of RASP patients operated were 1564 (min: 3 patients and maximum: 487 patients) from 2008 to 2020 (Table 1). The surgical approach was transperitoneal in 24 studies and extraperitoneal in 6 series (Table 1). All RASP cases were operated by da Vinci 4 arm Surgical Systems (Intuitive, Sunnyvale, CA, USA), except for Fareed et al. [18] and Steinberg et al. [34] who reported their initial experience with 8 and 10 cases of RASP using the da Vinci Single Port surgical systems (Intuitive, Sunnyvale, CA, USA). While Fareed et al. [18] performed their surgeries through a GelPort (Applied Medical, Rancho Santa Margarita, CA, USA) inserted directly into the bladder, Steinberg et al. [34] inserted their single port system extraperitoneally and performed their surgeries.

In all these studies, the mean operation time varied from 90 minutes to 274 minutes. The weight of the adenoma specimen varied between $46.4 \mathrm{gr}$ and $301 \mathrm{gr}$ (Table 1). The mean foley catheter removal time ranged from 1.6 days to 13 days. The mean hospital length of stay varied from 1 day to 8.8 days. In most series, although there were no intraoperative complications, perioperative complications up to $37.5 \%$ [18] were reported (Table 1). Estimated blood loss varied between $98 \mathrm{~mL}$ and $558 \mathrm{~mL}$. Transfusion rates were low. While in most series there was no need for blood transfusion, Fareed et al. [18] reported $87.5 \%$ transfusion rate in their study. The need of intraoperative conversion to open surgery was reported only in three studies $[15,37,39]$. Among them, only one study reported the reason of conversion to OSP. This was due to excessive bleeding $(500 \mathrm{~mL})$ and failure to progress during a RASP operation for a 260 gr prostatic adenoma [15].

Bladder neck contracture (BNC) was reported only in nine of these studies [12,13,22,28,37,40,42]. Although Yuh et al. [12] reported in one patient out of three and John et al. [13] in one patient out of 13, other researchers reported BNC in less than 1\%. Sorokin et al. [42] compared RASP and OSP and reported BNC in two and zero patients among OSP and RASP groups, respectively. Autorino et al. [37] in their comparative study reported BNC in three out of 487 patients $(0.6 \%)$ and three out of 843 patients $(0.35 \%)$ in RASP and LSP groups, respectively.

Urinary incontinence (UI) was reported in eight studies [15,21-23,34,38,40,44]. Although Nestler et al. [44] reported UI in nine out of 35 RASP patients (25.7\%), Elsamra et al. [23] in three out of 15 patients $(20 \%)$, and Steinberg et al. [34] in one out of 10 patients $(10 \%)$, in other series UI was less than $5.5 \%$ and mostly around $1 \%$. However, these UI rates are early UI rates with less than a 3-month follow-up.

Only 10 studies [15,21,22,28,30-32,37-39] reported on erectile function. In most of these studies, sexual function remained the same or improved (Table 1).

There are four studies comparing RASP outcomes to OSP [36,42-45] (Table 2). In all of these studies, the length of operation was significantly longer in RASP compared to OSP. On the other hand, RASP was found to have lower intraoperative EBL and shorter LOS. In addition, RASP was found to achieve similar functional outcomes, transfusion, and complication rates. 
Table 1. Robotic-assisted simple prostatectomy (RASP) series in the literature.

\begin{tabular}{|c|c|c|c|c|c|c|c|c|c|c|c|c|c|}
\hline Study & Study Type & Year & $\begin{array}{c}\text { Number of } \\
\text { Patients }\end{array}$ & Approach & $\begin{array}{c}\text { Prostate } \\
\text { Volume (mL) }\end{array}$ & $\begin{array}{c}\text { Operation Time } \\
(\min )\end{array}$ & $\begin{array}{l}\text { Conversion to } \\
\text { OSP }(\%)\end{array}$ & EBL $(\mathrm{mL})$ & $\begin{array}{c}\text { Transfusion Rate } \\
\quad(\%)\end{array}$ & $\begin{array}{l}\text { Specimen } \\
\text { Weight (g) }\end{array}$ & $\begin{array}{c}\text { Catheher } \\
\text { Removal Time } \\
\text { (Days) }\end{array}$ & $\begin{array}{c}\text { Length of } \\
\text { Stay (Days) }\end{array}$ & Complications $(\%)$ \\
\hline Sotelo et al. [3] & $\mathrm{NC}$ & 2008 & 7 & $\mathrm{~T}$ & 77.7 & 195 & 0 & 382 & 14.3 & 50.5 & 7.5 & 1.3 & 14 \\
\hline Yuh et al. [12] & $\mathrm{NC}$ & 2008 & 3 & $\mathrm{~T}$ & 323 & 211 & 0 & 558 & 33 & 301 & $\mathrm{NA}$ & 1.3 & 33 \\
\hline John et al. [13] & $\mathrm{NC}$ & 2009 & 13 & $\mathrm{E}$ & 100 & 210 & 0 & 500 & 0 & 82 & 6 & 6 & 7.7 \\
\hline Uffort et al. [14] & $\mathrm{NC}$ & 2010 & 15 & $\mathrm{~T}$ & 70.9 & 128.8 & 0 & 139.3 & 0 & 46.4 & 4.6 & 2.5 & 7 \\
\hline Sutherland et al. [15] & $\mathrm{NC}$ & 2011 & 9 & $\mathrm{~T}$ & 136.5 & 183 & 11.1 & 206 & 0 & 112 & 13 & 1.3 & 56 \\
\hline Vora et al. [17] & $\mathrm{NC}$ & 2012 & 13 & $\mathrm{~T}$ & 163 & 179 & 0 & 219 & 0 & 127 & 2.7 & 8.8 & 7.7 \\
\hline Fareed et al. [18] & $\mathrm{NC}$ & 2012 & 8 & $\mathrm{E}$ & 130 & 230 & 0 & 425 & 87.5 & 78 & 11 & 4.5 & 37.5 \\
\hline Matei et al. [19] & $\mathrm{NC}$ & 2012 & 35 & $\mathrm{~T}$ & 96.2 & 186 & 0 & 121 & 0 & 87 & 7.4 & 3.2 & 0 \\
\hline Coelho et al. [16] & $\mathrm{NC}$ & 2012 & 6 & $\mathrm{~T}$ & 157 & 90 & 0 & 208 & 0 & 145 & 4.8 & 1 & 0 \\
\hline Dubey et al. [35] & $\mathrm{NC}$ & 2012 & 3 & E & NA & 220 & 0 & 160 & $\mathrm{NA}$ & NA & 3 & 3.5 & $\mathrm{NA}$ \\
\hline Clavijo et al. [20] & $\mathrm{NC}$ & 2013 & 10 & $\mathrm{~T}$ & 81 & 106 & 0 & 375 & 10 & 81 & 8.9 & 1 & 20 \\
\hline Banapour et al. [10] & $\mathrm{NC}$ & 2014 & 16 & $\mathrm{~T}+\mathrm{E}$ & 141.8 & 228 & 0 & 197 & 0 & 94.2 & 8 & 1.3 & 12.5 \\
\hline Leslie et al. [21] & $\mathrm{NC}$ & 2014 & 25 & $\mathrm{~T}$ & 149.6 & 214 & 0 & 143 & 4 & 88 & 9 & 4 & 20 \\
\hline Stolzenburg et al. [24] & $\mathrm{NC}$ & 2014 & 10 & E & 143.9 & 122.5 & 0 & 228.8 & 0 & 102 & 7.4 & 8.4 & 10 \\
\hline Nestler et al. [22] & $\mathrm{NC}$ & 2014 & 18 & $\mathrm{~T}$ & 91 & 193 & 0 & 190 & 5.5 & 91 & 5.3 & $\mathrm{NA}$ & 5.5 \\
\hline Elsamra et al. [23] & $\mathrm{NC}$ & 2014 & 15 & $\mathrm{~T}$ & 156 & 189 & 0 & 290 & 0 & 110 & 8.67 & 2.4 & 14 \\
\hline Patel et al. [25] & $\mathrm{NC}$ & 2014 & 20 & $\mathrm{~T}+\mathrm{E}$ & NA & $\mathrm{NA}$ & $\mathrm{NA}$ & $\mathrm{NA}$ & $\mathrm{NA}$ & 134.7 & $\mathrm{NA}$ & 1.7 & $\mathrm{NA}$ \\
\hline Nething et al. [26] & $\mathrm{NC}$ & 2014 & 7 & $\mathrm{~T}$ & 144.9 & 204.7 & 0 & 521 & $\mathrm{NA}$ & 96.3 & 8.28 & 2.57 & 0 \\
\hline Autorino et al. [37] & C & 2015 & 487 & $\mathrm{~T}+\mathrm{E}$ & 110 & 154.5 & 3.1 & 200 & 1 & 75 & 7 & 2 & 16.6 \\
\hline Pokorny et al. [27] & $\mathrm{NC}$ & 2015 & 69 & $\mathrm{~T}$ & 129 & 97 & 0 & 200 & 1.5 & 84 & 3 & 4 & 33 \\
\hline Hoy et al. [36] & C & 2015 & 4 & $\mathrm{~T}$ & 238 & 161.3 & 0 & 218.8 & 0 & 123.6 & $\mathrm{NA}$ & 2.3 & 0 \\
\hline Castillo et al. [28] & $\mathrm{NC}$ & 2016 & 34 & $\mathrm{~T}$ & 117 & 96 & 0 & 200 & 5.8 & 76 & 4.6 & 2.2 & 20.5 \\
\hline Martin Garzon et al. [38] & $\mathrm{C}$ & 2016 & 154 & $\mathrm{E}$ & 78 & 157 & 0.7 & 455 & 6 & 71 & 9.3 & $\mathrm{NA}$ & 12 \\
\hline Pavan et al. [39] & $\mathrm{C}$ & 2016 & 130 & $\mathrm{~T}+\mathrm{E}$ & 118.5 & 150 & 10.8 & 250 & $\mathrm{NA}$ & 77 & 5 & 5 & 16.9 \\
\hline Umari et al. [40] & $\mathrm{C}$ & 2017 & 81 & $\mathrm{~T}$ & 130 & 105 & 0 & 250 & 1.2 & 89 & 3 & 4 & 31 \\
\hline Zhang et al. [41] & C & 2017 & 32 & E & NA & 274 & $\mathrm{NA}$ & $\mathrm{NA}$ & 9.4 & 110 & 8 & 2.3 & 3.1 \\
\hline Sorokin et al. [42] & $\mathrm{C}$ & 2017 & 59 & $\mathrm{~T}$ & 136.9 & 161.4 & 0 & 339 & 3.4 & 82.9 & 5.7 & 1.5 & 19 \\
\hline Cacciamani et al. [29] & $\mathrm{NC}$ & 2018 & 23 & $\mathrm{~T}$ & 108.1 & 160.6 & 0 & 98.6 & 0 & 63.1 & 7 & 2.1 & 4.3 \\
\hline Wang et al. [30] & $\mathrm{NC}$ & 2018 & 27 & $\mathrm{~T}$ & 82 & 169 & 0 & 235 & 0 & 47.5 & 1.6 & 3 & 22.2 \\
\hline Chavali et al. [31] & $\mathrm{NC}$ & 2018 & 28 & $\mathrm{~T}$ & 180 & 180 & 0 & 200 & 0 & 90 & 8 & 2 & 14 \\
\hline Johnson et al. [33] & $\mathrm{NC}$ & 2018 & 120 & $\mathrm{~T}$ & 121.5 & 157 & 0 & $\mathrm{NA}$ & 3.3 & 74 & 4 & 1 & 18.3 \\
\hline Nestler et al. [44] & C & 2019 & 35 & NA & 94.5 & 182 & 0 & $\mathrm{NA}$ & 9.4 & 77 & 5 & 5 & 25 \\
\hline Mourmouris et al. [43] & $\mathrm{C}$ & 2019 & 26 & $\mathrm{~T}$ & NA & 133.6 & 0 & 274 & 0 & 115 & 3 & 3.4 & 3.8 \\
\hline Simone et al. [32] & $\mathrm{NC}$ & 2019 & 12 & $\mathrm{~T}$ & 102 & 150 & 0 & 250 & 8 & 78 & 7 & 3 & 30 \\
\hline Steinberg et al. [34] & $\mathrm{NC}$ & 2019 & 10 & E & $\begin{array}{l}104 \\
\end{array}$ & $\begin{array}{l}72 \\
\end{array}$ & 0 & 141 & 0 & 65 & $\begin{array}{ll}1.9 \\
\end{array}$ & 1.1 & 0 \\
\hline
\end{tabular}


Table 1. Cont.

\begin{tabular}{|c|c|c|c|c|c|c|c|c|c|c|c|c|c|c|}
\hline Study & Study Type & $\begin{array}{c}\text { Preop } \\
\text { IPSS } \\
\end{array}$ & Postop IPSS & Preop QoL & Postop QoL & $\begin{array}{c}\text { Preop } \\
\text { IIEF/SHIM } \\
\end{array}$ & $\begin{array}{c}\text { Postop } \\
\text { IIEF/SHIM }\end{array}$ & $\begin{array}{c}\text { Preop Qmax } \\
\text { (mL/sn) }\end{array}$ & $\begin{array}{c}\begin{array}{c}\text { Postop Qmax } \\
\text { (mL/sn) }\end{array} \\
\end{array}$ & $\begin{array}{c}\text { Preop PVR } \\
(\mathrm{mL})\end{array}$ & Postop PVR (mL) & $\begin{array}{c}\text { Postop } \\
\text { İncontinence } \\
\end{array}$ & $\begin{array}{c}\text { Bladder neck } \\
\text { Contracture }(n)\end{array}$ & $\begin{array}{l}\text { Follow up } \\
\text { (Months) }\end{array}$ \\
\hline Sotelo et al. [3] & $\mathrm{NC}$ & 22 & 1.7 & 3.8 & 2.3 & NA & NA & 17.7 & 55.5 & NA & NA & NA & $\mathrm{N} / \mathrm{A}$ & 0 \\
\hline Yuh et al. [12] & $\mathrm{NC}$ & 17.7 & NA & 4.1 & NA & NA & NA & NA & $\mathrm{NA}$ & NA & $\mathrm{NA}$ & 0 & 1 & $\mathrm{NA}$ \\
\hline John et al. [13] & NC & $\mathrm{NA}$ & NA & NA & $\mathrm{NA}$ & $\mathrm{NA}$ & NA & NA & 23 & 85 & 0 & NA & 1 & 13 \\
\hline Uffort et al. [14] & $\mathrm{NC}$ & 23.9 & 1.8 & 4.9 & 2.2 & NA & NA & NA & NA & 265.8 & 44.2 & NA & $\mathrm{N} / \mathrm{A}$ & 3 \\
\hline Sutherland et al. [15] & $\mathrm{NC}$ & 17.8 & 7.8 & NA & $\mathrm{NA}$ & 12.7 (SHIM) & 12.5 (SHIM) & NA & NA & 214 & 18.2 & 1 & $\mathrm{~N} / \mathrm{A}$ & 9 \\
\hline Vora et al. [17] & $\mathrm{NC}$ & 18.2 & 5.3 & NA & NA & NA & NA & 4.4 & 19.1 & 207.3 & 12.7 & 0 & $\mathrm{~N} / \mathrm{A}$ & 7.2 \\
\hline Fareed et al. [18] & $\mathrm{NC}$ & 19.5 & 3 & NA & NA & NA & $\mathrm{NA}$ & 5.2 & 18 & 60 & 37 & 0 & $\mathrm{~N} / \mathrm{A}$ & 1 \\
\hline Matei et al. [19] & NC & 28 & 7 & NA & NA & NA & NA & 6.6 & 18.9 & NA & NA & NA & $\mathrm{N} / \mathrm{A}$ & 0 \\
\hline Coelho et al. [16] & $\mathrm{NC}$ & 19.8 & 5.5 & NA & NA & NA & NA & 7.7 & 19 & NA & NA & 0 & $\mathrm{~N} / \mathrm{A}$ & 2 \\
\hline Dubey et al. [35] & $\mathrm{NC}$ & $\mathrm{NA}$ & NA & NA & NA & NA & NA & NA & NA & NA & NA & NA & $\mathrm{N} / \mathrm{A}$ & $\mathrm{NA}$ \\
\hline Clavijo et al. [20] & NC & 18.8 & 1.7 & 3.7 & 0.5 & $\mathrm{NA}$ & NA & 12.4 & 33.5 & NA & NA & 0 & $\mathrm{~N} / \mathrm{A}$ & 1 \\
\hline Banapour et al. [10] & $\mathrm{NC}$ & 22 & 7 & 4 & 2 & NA & NA & NA & NA & 194 & 56 & 0 & $\mathrm{~N} / \mathrm{A}$ & 0 \\
\hline Leslie et al. [21] & $\mathrm{NC}$ & 23.9 & 3.6 & $\mathrm{NA}$ & $\mathrm{NA}$ & 12.8 (SHIM) & NA & 11.3 & 20 & 208.1 & 36.9 & 1 & $\mathrm{~N} / \mathrm{A}$ & 6 \\
\hline Stolzenburg et al. [24] & NC & 21.9 & 3.4 & NA & NA & NA & NA & 9.3 & 20.7 & 121.9 & 57.5 & 0 & N/A & 6 \\
\hline Nestler et al. [22] & $\mathrm{NC}$ & 25 & 6.1 & 5 & 1.1 & 59 (IIEF) & 56.8 (IIEF) & 9 & 28.2 & NA & NA & 1 & 0 & 1 \\
\hline Elsamra et al. [23] & $\mathrm{NC}$ & 16.2 & 4.5 & NA & NA & $\mathrm{NA}$ & $\mathrm{NA}$ & NA & $\mathrm{NA}$ & 428 & 33 & 3 & $\mathrm{~N} / \mathrm{A}$ & 3 \\
\hline Patel et al. [25] & $\mathrm{NC}$ & 14.7 & NA & NA & $\mathrm{NA}$ & $\mathrm{NA}$ & NA & NA & NA & 414 & NA & NA & $\mathrm{N} / \mathrm{A}$ & NA \\
\hline Nething et al. [26] & $\mathrm{NC}$ & $\mathrm{NA}$ & NA & NA & NA & NA & NA & NA & NA & NA & NA & 0 & $\mathrm{~N} / \mathrm{A}$ & 10 \\
\hline Autorino et al. [37] & $\mathrm{C}$ & 23 & 7 & 4 & NA & 15 (SHIM) & 15 (SHIM) & 8 & 25 & 108 & NA & NA & 3 patients & 12 \\
\hline Pokorny et al. [27] & $\mathrm{NC}$ & 25 & 3 & $\mathrm{NA}$ & NA & NA & NA & 7 & 23 & 73 & 0 & 0 & $\mathrm{~N} / \mathrm{A}$ & 6 \\
\hline Hoy et al. [36] & $\mathrm{C}$ & $\mathrm{NA}$ & NA & NA & NA & NA & $\mathrm{NA}$ & $\mathrm{NA}$ & $\mathrm{NA}$ & NA & NA & NA & $\mathrm{N} / \mathrm{A}$ & 3 \\
\hline Castillo et al. [28] & $\mathrm{NC}$ & 23.5 & 7.1 & NA & NA & NA & NA & 10.4 & 23.1 & NA & NA & 0 & 1 patient & 12 \\
\hline Martin Garzon et al. [38] & C & 22 & 6.5 & 3.8 & 1 & 18.5 (SHIM) & 16 (SHIM) & 11.5 & 33 & NA & NA & 9 & $\begin{array}{c}1 \text { patient developed } \\
\text { anterior urethral } \\
\text { stricture }\end{array}$ & 12 \\
\hline Pavan et al. [39] & $\mathrm{C}$ & 23 & 5 & 6 & NA & 18 (SHIM) & 17 (SHIM) & 9 & 22 & NA & NA & NA & $\mathrm{N} / \mathrm{A}$ & 10.3 \\
\hline Umari et al. [40] & $\mathrm{C}$ & 25 & 5 & NA & $\mathrm{NA}$ & $\mathrm{NA}$ & NA & 8 & 23 & 73 & 0 & 1 & 0 & 12 \\
\hline Zhang et al.[41]] & C & $\mathrm{NA}$ & NA & NA & $\mathrm{NA}$ & NA & NA & NA & NA & $\mathrm{NA}$ & NA & NA & $\mathrm{N} / \mathrm{A}$ & NA \\
\hline Sorokin et al. [42] & C & 18.8 & 7.3 & 3.9 & 1.3 & NA & NA & 9.8 & 22.4 & 118 & 3.5 & 0 & 0 & 6 \\
\hline Cacciamani et al. [29] & $\mathrm{NC}$ & 23.1 & NA & NA & NA & NA & NA & NA & NA & NA & NA & 0 & $\mathrm{~N} / \mathrm{A}$ & 3 \\
\hline Wang et al. [30] & $\mathrm{NC}$ & 25 & NA & 6 & NA & 18 (IIEF) & 17.5 (IIEF) & 6 & NA & 85 & NA & 0 & $\begin{array}{c}\text { No strictue but } 7 \\
\text { patients required } \\
\text { closure of small } \\
\text { urethrotomies } \\
\end{array}$ & 16.4 \\
\hline Chavali et al. [31] & $\mathrm{NC}$ & 19 & NA & NA & NA & NA & NA & 9 & NA & 120 & NA & NA & $\mathrm{N} / \mathrm{A}$ & NA \\
\hline Johnson et al. [33] & $\mathrm{NC}$ & $\mathrm{NA}$ & NA & NA & $\mathrm{NA}$ & $\mathrm{NA}$ & NA & 8.9 & 18.8 & NA & 9 & 0 & $\mathrm{~N} / \mathrm{A}$ & 3.2 \\
\hline Nestler et al. [44] & $\mathrm{C}$ & 23 & NA & 5 & NA & NA & NA & NA & NA & NA & NA & 0 & $\mathrm{~N} / \mathrm{A}$ & 12 \\
\hline Mourmouris et al. [43] & $\mathrm{C}$ & 22.9 & 5.7 & NA & NA & NA & NA & 10.1 & 19.1 & 178.5 & 25.5 & NA & $\mathrm{N} / \mathrm{A}$ & 3 \\
\hline Simone et al. [32] & $\mathrm{NC}$ & 33 & 6 & NA & NA & 27 (IIEF) & 27 (IIEF) & 7.7 & 18.6 & 175 & 30 & 0 & N/A & 12 \\
\hline Steinberg et al. [34] & $\mathrm{NC}$ & 20.8 & 12.9 & NA & NA & NA & NA & 8.6 & 11.2 & 119 & 40.9 & 1 (transient) & $\mathrm{N} / \mathrm{A}$ & 28.7 \\
\hline
\end{tabular}

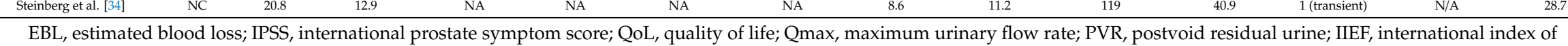
erectile function; SHIM, Sexual Health Inventory for Men; NC, non-comparative; C, comparative. 
Table 2. RASP versus open simple prostatectomy (OSP) and minimally invasive simple prostatectomy comparative studies.

\begin{tabular}{|c|c|c|c|c|c|}
\hline Reference & Versus & Study Design & Number of Cases & Baseline Characteristics & Main Findings \\
\hline Pavan et al. [39] & LSP & Multileft retrospective & $\begin{array}{l}319 \\
(\mathrm{LSP}=189 ; \text { RASP }=130)\end{array}$ & $\begin{array}{l}\text { Median prostate volume larger for RASP } \\
(118.5 \text { versus } 109 \mathrm{~mL} ; p=0.02)\end{array}$ & $\begin{array}{l}\text {-No significant difference for blood loss, catheter time, hospital stay, } \\
\text { major complication rate } \\
\text {-On MVA technique not influencing 'trifecta' outcome }\end{array}$ \\
\hline Martin Garzon et al. [38] & LSP & Single left retrospective & $\begin{array}{l}315 \\
(\mathrm{LSP}=82 ; \mathrm{IF}-\mathrm{RASP}=75)\end{array}$ & No differences & -Similar surgical outcomes and functional outcomes at 1 year \\
\hline Umari et al. [40] & HoLEP & Single left retrospective & $\begin{array}{l}126 \\
(\text { HoLEP }=45 ; \text { RASP }=81)\end{array}$ & $\begin{array}{l}\text { RASP patients younger (median age } 69 \\
\text { versus } 74, p=0.032) \text {, less healthy } \\
\text { (Charlson index }>2 \text { in } 62 \% \text { versus } 29 \% \\
p<0.001), \text { with higher preoperative IPSS } \\
(25 \text { versus } 21, p=0.049)\end{array}$ & $\begin{array}{l}\text {-Similar improvement for Qmax, PVR, IPSS } \\
\text {-Similar operative time } \\
\text {-Catheter time ( } 3 \text { versus } 2, p=0.005 \text { ) and hospital stay ( } 4 \text { versus } 2 \text { days, } \\
p=0.0001) \text { longer for RASP } \\
\text {-Complication rates similar }\end{array}$ \\
\hline Zhang et al. [40] & HoLEP & Bileft retrospective & $\begin{array}{l}632 \\
(\text { HoLEP }=600 ; \text { RASP }=32)\end{array}$ & No differences & $\begin{array}{l}\text {-Mean operative time shorter for HoLEP (103 versus } 274 \mathrm{~min}, p<0.001 \\
\text {-HoLEP with lower transfusion rate (1.8 versus } 9.4 \%, p=0.03) \text {, shorter catheter } \\
\text { time }(0.7 \text { versus } 8 \text { days, } p<0.001) \text {, and shorter hospital stay ( } 1.3 \text { versus } \\
2.3 \text { days, } p<0.001) \\
\text {-Complication rates similar }\end{array}$ \\
\hline Sorokin et al. [42] & OSP & $\begin{array}{l}\text { Single left retrospective } \\
\text { propensity score matched }\end{array}$ & $\begin{array}{l}188 \\
(\mathrm{OSP}=59 ; \mathrm{RASP}=59)\end{array}$ & No differences & $\begin{array}{l}\text {-RASP with shorter mean hospital stay }(1.5 \text { versus } 2.6 \text { days, } p<0.001) \text {, } \\
\text { but longer operative time }(161 \text { versus } 93 \mathrm{~min}, p<0.001) \\
\text {-Lower blood loss ( } 339 \text { versus } 587 \mathrm{~mL}, p<0.001) \text { and hemoglobin drop } \\
\text { (12.3\% versus } 19.5 \%, p=0.001 \text { ) for RASP } \\
\text {-No differences in transfusion rates, functional outcomes, complication rate }\end{array}$ \\
\hline Mourmouris et al. [43] & OSP & Bileft prospective & $\begin{array}{l}41 \\
(\mathrm{OSP}=15 ; \mathrm{RASP}=26)\end{array}$ & $\begin{array}{l}\text { RASP patients younger (median age } 66.73 \\
\text { versus } 70.46 p=0.032 \text { ) }\end{array}$ & $\begin{array}{l}\text {-RASP achieves similar functional outcomes and provides significant } \\
\text { advantages, such as decreased blood loss, faster catheter removal (because of } \\
\text { the uneventful postoperative course), a shorter LOS and a lower complication } \\
\text { rate, at the cost of a longer operating time }\end{array}$ \\
\hline Nestler et al. [44] & OSP, ThuVEP & $\begin{array}{l}\text { Multileft, } \\
\text { Matched Pair Analysis }\end{array}$ & $\begin{array}{l}105 \\
(\text { OSP = 35; RASP = 35; } \\
\text { ThuVEP = 35) }\end{array}$ & No differences & $\begin{array}{l}\text {-Blood loss in OSP was significantly higher compared to the minimal invasive } \\
\text { approaches. ThuVEP showed a median operation time of } 83 \text { min and was } \\
\text { therefore significantly faster than OSP with } 130 \mathrm{~min}(p=0.004) \text { and RASP } \\
\text { needing } 182 \text { min. Significant advantages for the minimal invasive approaches } \\
\text { compared to open surgery concerning blood loss, transfusion rates and } \\
\text { early continence }\end{array}$ \\
\hline Autorino et al. [37] & LSP & $\begin{array}{l}\text { Multileft, } \\
\text { Retrospective }\end{array}$ & $\begin{array}{l}1330 \\
(\text { RASP }=487 ; \text { LSP = 843) }\end{array}$ & $\begin{array}{l}\text { Median Charlson Index for LSP patients: } \\
4 \text { and for RASP patients: } 2 \\
\text { Median prostate volume is larger in RASP } \\
\text { patients ( } 110 \text { versus } 99 \mathrm{~mL})\end{array}$ & $\begin{array}{l}\text { Trifecta outcome, arbitrarily defined as a combination of the following } \\
\text { postoperative events: International Prostate Symptom Score }<8 \text {, maximum } \\
\text { flow rate }>15 \mathrm{~mL} / \mathrm{s} \text {, and no perioperative complications. Trifecta outcome was } \\
\text { not significantly influenced by the type of procedure (robotic versus } \\
\text { laparoscopic; } p=0.136 ; \text { odds ratio: } 1.6 ; 95 \% \text { confidence interval, } 0.8-2.9 \text { ), } \\
\text { whereas operative time }(p=0.01 ; \text { OR: } 0.9 ; 95 \% \text { CI, } 0.9-1.0 \text { ) and estimated blood } \\
\text { loss }(p=0.03 ; \mathrm{OR}: 0.9 ; 95 \% \mathrm{CI}, 0.9-1.0 \text { ) were the only two significant factors. }\end{array}$ \\
\hline Hoy et al. [36] & OSP & $\begin{array}{l}\text { Single left } \\
\text { Retrospective }\end{array}$ & $\begin{array}{l}32 \\
(\text { RASP }=4 ; \text { OSP }=28)\end{array}$ & $\begin{array}{l}\text { No differences } \\
\text { RASP patients younger (median age } 69.3 \\
\text { versus } 75.18, p=0.17 \text { ), } \\
\text { Prostate volume on TRUS }(\mathrm{mL}) \\
\text { RASP }=239 \pm 49.8 \\
\text { OSP }=180 \pm 54.70 .09\end{array}$ & $\begin{array}{l}\text {-There was a significant difference in the mean length of operation, with RASP } \\
\text { exceeding OSP (161 versus } 79 \mathrm{~min} ; p=0.008) \text {. } \\
\text {-The mean intraoperative blood loss was significantly higher in the open } \\
\text { group }(835.7 \text { versus } 218.8 \mathrm{~mL} ; p=0.0001) \text {. } \\
\text {-Mean LOS was shorter in the RASP group ( } 2.3 \text { versus } 5.5 \text { days; } p=0.0001) \text {. } \\
\text {-No significant differences were noted in the } 90 \text {-day transfusion rate }(p=0.13) \text { ) } \\
\text { or overall complication rate at } 0 \% \text { with RASP versus } 57.1 \% \text { with OSP }(p=0.10)\end{array}$ \\
\hline
\end{tabular}

HoLEP, Holmium laser enucleation of the prostate, ThuVEP: Thulium laser VapoEnucleation, IF-RASP, intrafascial robotic assisted simple prostatectomy, LSP, laparoscopic simple prostatectomy, MVA, multivariable analysis, OSP, open simple prostatectomy, PVR, postvoid residual. 
Similarly, in three studies [37-39] RASP was compared to LSP. While two studies [38,39] reported surgical and functional outcomes with no significant difference for EBL, catheter removal time, LOS, major complications rate between two groups, Autorino et al. [37] reported shorter operative time for LSP but less EBL in favor of RASP.

There were two studies [40,41] comparing HoLEP to RASP and one study [44] comparing ThuVEP to RASP. While Umari et al. [40] reported similar improvements for Qmax, post-voiding residual urine (PVR), IPSS, similar operative time, and complication rates (no BNC observed in both groups), Zhang et al. [41] reported shorter mean operative time, catheter removal time, LOS, and lower transfusion rates in favor of HoLEP. Complication rates were also similar in this study.

Nestler et al. [44] reported a matched pair analysis results of comparison for OSP, ThuVEP, and RASP. In their study, ThuVEP showed significantly lower operation time, blood loss, and transfusion rates over OSP $(p<0.01)$. However, although operation time was significantly lower in ThuVEP than RASP, the difference in favor of ThuVEP for blood loss and transfusion rates were not statistically significant ( $p=0.18$ and $p=0.36$, respectively). Postoperative Qmax, IPSS, and QoL questionnaires improved without significant difference between the approaches $(p>0.08)$. In their study, only five patients needed one pad during the first $24 \mathrm{~h}$ after ThuVEP and median pad use was zero in ThuVEP. After RASP, 26 out of 35 patients reported no incontinence. Of the remaining nine patients, six patients needed one pad, two patients needed two pads, and one patient needed three pads per day. The difference between these two groups however was not statistically significant. In OSP, median pad use was 1 and mean pad use was 1.23, but overall 28 out of 35 patients needed pads, 14 patients one pad, 12 patients two pads, and 2 patients three pads. Mean as well as median pad use were significantly higher in OSP compared to the minimal invasive approaches $(p \leq 0.001)$.

\section{Discussion}

Since 2008, when RASP was first described by Sotelo et al. [3], the procedure has been increasingly performed worldwide. Parallel to the increase in the centers implementing this technique, the literature on RASP also showed a steady growth with encouraging results [46]. RASP can be classified as transcapsular or transvesical. The approach can be transperitoneal or extraperitoneal. The cystostomy incision can be made horizontal or vertical. Recently Castillo et al. [28] reported a modified technique with longitudinal capsular and vesical incision, plication of prostatic capsule, and a posterior only urethrovesical anastomosis. Clavijo et al. [20] developed and reported a new "intrafascial technique" for RASP which was developed on the same principles of intrafascial radical prostatectomy technique. They performed near-complete excision of all prostatic tissue with preserving the seminal vesicles, periprostatic fascia, and puboprostatic ligaments. A subsequent vesicourethral anastomosis was performed in a manner similar to that of robotic prostatectomy. They claimed that this technique minimizes postoperative hematuria and has the advantage of not requiring postoperative bladder irrigation. Martin Garzon et al. [38] analyzed 236 minimally invasive simple prostatectomies. They reported 82 laparoscopic and 79 RASP with Millin approach and 75 RASP with intrafascial approach. They found similar surgical outcomes including complication rate, transfusion rate, and functional outcomes for IPSS, SHIM, continence, and Qmax between three procedures. They also concluded that an intrafascial technique does not require postoperative irrigation. Cacciamani et al. [29] reported RASP with $360^{\circ}$ circumferential reconstruction and Wang et al. [30] published "Urethra Sparing" RASP series based on "Madigan surgical technique" first described in 1990 by Dixon et al. [47]. Recently, Simone et al. [32] reported, "Urethra and ejaculation preserving RASP" by implementing near-infrared fluorescence imaging-guidance to Madigan technique. They reported a 1-yr follow-up, median IPSS score, IIEF score, and MSHQ-EjD Short Form score as 5 (IQR 4-8), 26 (IQR 26-28), and 12 (IQR 1-14), respectively. They achieved satisfactory anterograde ejaculation in $66 \%$ of their patients.

Although there is no prospective randomized study in the current literature, the data of existing case series (Table 1) and comparative studies (Table 2) suggest that RASP is a safe and efficacious procedure in the treatment of BPH patients with $>80 \mathrm{gr}$ of prostate size. In these studies, RASP reported 
to have longer operation time than OSP, LSP, and laser enucleations. However, RASP achieved similar functional outcomes for IPSS, PVR, Qmax, QoL, EBL, LOS, transfusion, and complication rates. Parsons et al. [45] studied the Nationwide Inpatient Sample (NIS) from 1998 to 2010 and identified 34,418 and 193 patients undergoing OSP and MISP, respectively. They focused on 2008 to 2010 because of the trend shift observed (increase in MISP and decrease in OSP) in surgical techniques during the last years. In their final analysis, 6027 OSP and 182 MISP patients were evaluated. They reported that MISP patients were more likely to have higher Charlson comorbidity scores and less likely to undergo transfusion, but these differences did not attain significance. They also found no significant differences in median LOS, hospital charges, or unadjusted in-hospital mortality. Similarly, Lucca et al. [48] performed a systematic review and meta-analysis comparing outcomes of MISP with OSP. They included 764 patients and 27 observational studies in which 8 were robotic surgery with 119 patients. They reported similar improvements in Qmax, IPSS in both groups while MISP group had significantly lower LOS, length of catheter use and EBL than OSP. However, the duration of operation was significantly lower in OSP. Authors, unfortunately, did not analyze RASP, LSP, and OSP subgroups separately. Li et al. [49] also conducted a systematic review and meta-analysis with 10 studies including 995 patients. Six of the studies were comparisons between LSP and OSP, while four were comparisons between RASP and OSP. They found no significant differences between MISP and OSP regarding postoperative IPSS, QoL, Qmax, PVR, and irrigation time. MISP group was found to have lesser EBL, shorter catheterization time, shorter LOS, lower transfusion rate, and lower complication rates compared with OSP group. They also reported longer operation time in MSP group. Moreover, they performed a subgroup analysis between RASP, LSP, and OSP to detect the possible differences. They demonstrated that OT, EBL, LOS, transfusion rate, and the number of complications in the LSP group was less than that in the OSP group (OR: $0.52 ; 95 \%$ CI: 0.37 to $0.74 ; p=0.002$ ), and the RASP group had the same result (OR: $0.42 ; 95 \%$ CI: 0.23 to $0.77 ; p=0.005)$. These results were in conjunction with the results of our systematic review.

Autorino et al. [37] collected data from 23 centers worldwide and reported the outcomes of largest RASP series to date. They reported 487 cases with median operative time of 154 min, median EBL of $200 \mathrm{~mL}$, and median 2 days LOS. The time to foley removal was 7 (5-9) days in the study. However, since most patients were discharged from the hospital with their foley catheters and were removed on outpatient follow-up visits, the catheter removal time was not a good indicator for the evaluation of RASP outcomes. Similarly, blood transfusion rates were also not a good indicator for evaluation of RASP outcomes since they vary among institutions, according to patients' comorbidities and surgeons' preference.

Umari et al. [40] compared 81 RASP to 45 HoLEP procedures and reported no difference in terms of operative time and complications. On the other hand, Zhang et al. [41] compared outcomes of 32 RASP and 600 HoLEP and reported shorter operative time in favor of HoLEP (103 min (std:47) versus 274 min (std: 49) $(p<0.001)$ ). They collected HoLEP data from different centers. Johnson et al. [33] conducted a study to determine the learning curve for RASP and reported that 10-12 cases may be required for somebody with robotic experience to become proficient for RASP. On the other hand, Brunckhorst et al. [50] estimated 40-60 cases to be enough for HoLEP surgery. The authors concluded that learning curve might have an impact and favor RASP over HoLEP. Nestler et al. [44] reported significantly shorter operation time in ThuVEP than RASP (83 min versus $182 \mathrm{~min}$, respectively). Regarding discrepancies in operating times among RASP series, Johnson et al. [33] also reported that operative time improved at a rate of 1 to 2 minutes per case, which represented the gaining experience of the surgery. Besides, the previous total robotic surgeries and robotic lower urinary system operations, like radical prostatectomies, cystectomies, and robotic systems (4 arm si or xi Da Vinci or single port system Da Vinci ), might have an impact on RASP operation time as well. Leonardo et al. [51] recently published a systematic review including 9 randomized clinical trials with most of them reporting data at 1 year to assess the management of patients with big prostates $(>80 \mathrm{cc})$. The investigated trials compared enucleation, vaporization, open techniques such as transurethral 
resection in saline (TURis), transurethral vaporization in saline (TUVis), bipolar plasma enucleation of prostate (BPEP), plasma kinetic resection of prostate (PKRP), transurethral vaporization of prostate (TVP), transurethral enucleation and resection of prostate (TUERP), plasma kinetic enucleation of prostate (PKEP), photo vaporization of prostate (PVP), diode enucleation of prostate (DILEP), HoLEP, ThuVEP, and OSP. In terms of perioperative outcomes, all the techniques had similar operative times and resected prostate weight, whereas, catheterization time and hospital stay were better in endoscopic techniques compared to open surgery. In terms of functional outcomes (IPSS, QMAX, and PVR), none of the techniques were proven superior to the other. When considering complications, open procedures carried a higher risk of transfusions, while no technique was proven superior to the others in terms of transient urge UI, BNC, and reintervention.

BNC was reported as 3.0\% $\pm 0.2 \%$ (between $0 \%$ and $6 \%$ ) in OSP series [52]. This systematic review showed that BNC was reported $<1 \%$ in most published RASP series. Although current data does not allow to metaanalyze the results of these studies because of uniformity of surgical techniques and data itself, one may speculate that BNC occurs less when compared to OSP and LSP. There is no data for comparing RASP with laser techniques as well. Leonardo et al. [51], in their recent systematic review, found the rate of BNC was between $15 \%$ and $75 \%$ in OSP and other endoscopic enucleation techniques, and they stated that none of the studies was designed to prove differences in this term. Similarly, UI was reported $<5.5 \%$ in most RASP series. However, follow-up was very short and type of incontinence (stress versus urgency) was not specified in most series. The rate of urge UI was reported between $5 \%$ and $30 \%$ in OSP, endoscopic, and vaporization techniques [51]. Unfortunately, none of the current studies specifically addressed the comparative data of RASP and laser surgeries on the incidence of irritative symptoms that might be seen especially after laser surgeries in early postoperative period. Regarding erectile functions, current data does not allow to make direct comparison between RASP, LSP, OSP, and other endoscopic techniques. At least in most of the studies, which report on erectile or sexual function, postoperative results seemed unchanged or even increased in some extent. Long term RCT's are needed to shed light on these gray zones.

Transurethral resection of prostate (TUR-P) was widely used for the treatment of symptomatic $\mathrm{BPH}$ even for $>80 \mathrm{gr}$ prostates. In the current literature, there were studies comparing effectiveness between OSP and bipolar TUR-P [53]; however, to date, there was no study which compares the outcomes of bipolar TUR-P and RASP for the treatment of prostate glands $>80 \mathrm{gr}$. One might speculate that bipolar TUR-P is the real competitor of the RASP even in cases of high prostatic volume, primarily due to the quicker and similar amount of tissue resection. Reviewing the literature, Zhu et al. [54] in 52 out of 132 patients who underwent bipolar TUR-P with preoperative transrectal ultrasound measured prostate volume of $>80 \mathrm{~mL}$ (mean $101.6 \mathrm{~mL}$ ) and reported that the resected BPH tissue in that specific subgroup was $64.75 \%$ of the initial measured prostate volume. Similarly, Matei et al. [19] reported in 25 patients with the mean (range) transrectal ultrasound (TRUS) prostate volume of 93.4 (70-150) $\mathrm{mL}$, and the mean volume of tissue removed was $63.8 \mathrm{~mL}$, accounting for $68.3 \%$ of the initial prostate volume. When they stratified patients into three subgroups, patients with prostate volumes of $<80 \mathrm{~mL}$, $81-99 \mathrm{~mL}$, and $>100 \mathrm{~mL}$, the percentage of prostatic tissue removed decreased from $71.2 \%$ to $69 \%$ to $66.5 \%$, respectively. Ou et al. [55] compared TUR-P and OSP and reported similar results in their series of 69 patients where only $53.2 \%$ of prostatic tissue was resected in the TURP group compared with $84.4 \%$ in the OSP group. Bach et al. [56] compared the efficacy of TUR-P, ThuVEP, and Greenlight PVP in 2648 patients with BPH. They reported efficacy, as measured in resection weight per minute of total operating time, was higher in ThuVEP than in TUR-P, independent of prostate volume. The tissue removal per operating time was higher in ThuVEP even in prostates $<40 \mathrm{cc}(15.4 \%)$, and this efficiency increased with increasing prostate volume to $51.6 \%$ in prostates larger than $80 \mathrm{cc}$. On the other hand, $\mathrm{Ou}$ et al. [53] in a prospective randomized study with $98 \mathrm{BPH}$ patients compared the effectiveness of TUERP to OSP for prostate volumes of $>80 \mathrm{~mL}$. They reported the resected adenoma weight in the OSP group was more than that in the TUERP group, but the difference was not statistically significant $(p=0.062)$. When postoperative PSA reduction was used as a surrogate marker of adequacy of tissue 
removal, at 12 months postoperatively, a decrease in PSA was $72.9 \%$ (from 5.9 to $1.6 \mathrm{ng} / \mathrm{mL}$ ) and $78.6 \%$ (from 5.6 to $1.2 \mathrm{ng} / \mathrm{mL}$ ) in patients who underwent TUERP and OSP group, respectively. The mean postoperative PSA reductions in each group were similar $(p=0.12)$. The mean weight of the adenoma specimen removed in RASP series varied between $46.4 \mathrm{gr}$ and $301 \mathrm{gr}$ and was accounting for $50 \%$ to $100 \%$ of preoperatively measured prostatic adenoma tissue. Matei et al. [19] after reviewing the RASP series reported that the relative amounts of removed tissue was higher, both when mean values (81.2\% for RASP versus $75.6 \%$ for OSP and $70.4 \%$ for TUR) and median values were considered $(68.6 \%$ for RASP versus $65.7 \%$ for OSP and $66.7 \%$ for TUR). Jones et al. [57] conducted a systematic review and meta-analysis comparing HoLEP with SP, including OP, RASP, and LSP. Only three randomized studies (263 patients) were included among the evaluated articles. Each trial compared HoLEP with OP. No studies were identified, which compared HoLEP with RASP or LSP directly. The mean prostate volume was $114 \mathrm{~mL}$ in the HoLEP group and $119 \mathrm{~mL}$ in the simple prostatectomy group. They reported that OSP was associated with a significantly shorter operative time and greater tissue removal. Nevertheless, further studies were still required to reveal unanswered questions about these issues.

Cost was another controversial topic and should be taken into account. Cost-benefit analysis was a complex problem and affected by multiple factors such as hospital costs, complications, and reimbursement issues which vary significantly between countries and healthcare systems [58]. Sutherland et al. [15] reported that RASP was expectedly expensive compared to OSP, adding an average of USD 2797 to the operating charges, which does not include the initial investment cost of the robotic system. On the other hand, Matei et al. [19] noted that considering the cost of hospitalization, transfusion rates and need for continuous bladder irrigation, RASP may become cheaper overall than OSP and has similar cost to bipolar TUR-P. Pariser et al. [59] found a significant difference in hospital charges related to the presence of complications after SP. While patients with complications cost USD 51,295 , patients without a complication cost USD 32,305. Considering that RASP has lower complication rates compared to OSP, one might assume that RASP may cost less overall. Salonia et al. [60] compared the cost of OSP and HoLEP and reported average cost of HoLEP was EUR 2356 which was $9.6 \%$ less than OSP. The usage of robotic instruments during RASP differs among surgeons and institutions. Since most instruments were used a maximum of 10 times and each instrument costs USD 2500, using extra arms may increase the cost of RASP. In this regard, the surgeon's experience and the technique impacts the cost of RASP. Still, more studies are needed to establish the recent and actual cost of RASP. Considering all these cost issues and the longer operation time with RASP, reserving RASP for patients with concomitant bladder stones of considerable size or diverticula, which can be treated simultaneously, is logical [44]. Considering the RASP learning curve, which was at least 10-15 cases, if the caseload of RASP per center is less than 10-15 per year, implementation of RASP should be debated because of aforementioned reasons [44]. Yet, well-designed studies are needed before a clear conclusion can be drawn.

\section{Conclusions}

For patients with LUTS who need surgery with prostate glands $>80 \mathrm{gr}$, RASP is a good alternative MIS, especially when laser systems or surgical skills are unavailable. This systematic review indicated that RASP does not only provide similar improvements in functional outcomes for IPSS, PVR, Qmax, QoL, but also has similar complication rates, EBL, LOS to OSP, ThuVEP, and HoLEP. While further studies are needed on cost analysis, learning curve, and best surgical approach, implementation of RASP in centers with established robotic programs are becoming attractive and increasing every day. Nevertheless, prospective RCT's with multicenter and large sample sizes are needed to confirm the results of this systematic review. 
Author Contributions: Conceptualization, Y.K., D.B., M.P.L. and J.d.1.R.; methodology, Y.K., A.E.C.; software, E.K.; validation, Y.K., A.E.C. and D.B.; formal analysis, A.E.C., E.K. and Y.K.; investigation, Y.K., A.E.C., D.B.; resources, A.E.C., D.B., Y.K.; data curation, D.B., Y.K., E.K.; writing-original draft preparation, Y.K.; writing-review and editing, D.B., J.d.1.R.; visualization, M.P.L.; supervision, D.B. and J.d.1.R.; project administration, A.E.C.; funding acquisition, none. All authors have read and agreed to the published version of the manuscript.

Funding: This research received no external funding.

Conflicts of Interest: The authors declare no conflict of interest.

\section{References}

1. Elshal, A.M.; El-Nahas, A.R.; Barakat, T.S.; Elsaadany, M.M.; El-Hefnawy, A.S. Transvesical open prostatectomy for benign prostatic hyperplasia in the era of minimally invasive surgery: Perioperative outcomes of a contemporary series. Arab. J. Urol. 2013, 11, 362-368. [CrossRef] [PubMed]

2. Mariano, M.B.; Graziottin, T.M.; Tefilli, M.V. Laparoscopic prostatectomy with vascular control for benign prostatic hyperplasia. J. Urol. 2002, 167, 2528-2529. [CrossRef]

3. Sotelo, R.; Clavijo, R.; Carmona, O.; Garcia, A.; Banda, E.; Miranda, M.; Fagin, R. Robotic simple prostatectomy. J. Urol. 2008, 179, 513-515. [CrossRef] [PubMed]

4. Meyer, D.; Weprin, S.; Zukovski, E.B.; Porpiglia, F.; Hampton, L.J.; Autorino, R. Rationale for Robotic-assisted Simple Prostatectomy for Benign Prostatic Obstruction. Eur. Urol. Focus 2018, 4, 643-647. [CrossRef]

5. Foster, H.E.; Barry, M.J.; Dahm, P.; Gandhi, M.C.; Kaplan, S.A.; Kohler, T.S.; Lerner, L.B.; Lightner, D.J.; Parsons, J.K.; Roehrborn, C.G.; et al. Surgical Management of Lower Urinary Tract Symptoms Attributed to Benign Prostatic Hyperplasia: AUA Guideline. J. Urol. 2018, 200, 612-619. [CrossRef]

6. Gratzke, C.; Bachmann, A.; Descazeaud, A.; Drake, M.J.; Madersbacher, S.; Mamoulakis, C.; Oelke, M.; Tikkinen, K.A.O.; Gravas, S. EAU Guidelines on the Assessment of Non-neurogenic Male Lower Urinary Tract Symptoms including Benign Prostatic Obstruction. Eur. Urol. 2015, 67, 1099-1109. [CrossRef]

7. Vincent, M.W.; Gilling, P.J. HoLEP has come of age. World J. Urol. 2015, 33, 487-493. [CrossRef]

8. Placer, J.; Gelabert-Mas, A.; Vallmanya, F.; Manresa, J.M.; Menendez, V.; Cortadellas, R.; Arango, O. Holmium laser enucleation of prostate: Outcome and complications of self-taught learning curve. Urology 2009, 73, 1042-1048. [CrossRef]

9. Robert, G.; Cornu, J.N.; Fourmarier, M.; Saussine, C.; Descazeaud, A.; Azzouzi, A.R.; Vicaut, E.; Lukacs, B. Multicentre prospective evaluation of the learning curve of holmium laser enucleation of the prostate (HoLEP). BJU Int. 2016, 117, 495-499. [CrossRef]

10. Banapour, P.; Patel, N.; Kane, C.J.; Cohen, S.A.; Parsons, J.K. Robotic-assisted simple prostatectomy: A systematic review and report of a single institution case series. Prostate Cancer Prostatic Dis. 2014, 17, 1-5. [CrossRef]

11. Robotic-Assisted Robotic Adenomectomy: A Systematic Review. Available online: https://osf.io/kdf7p/ (accessed on 6 April 2020).

12. Yuh, B.; Laungani, R.; Perlmutter, A.; Eun, D.; Peabody, J.O.; Mohler, J.L.; Stricker, H.; Guru, K.A. Robot-assisted Millin's retropubic prostatectomy: Case series. Can. J. Urol. 2008, 15, 4101-4105.

13. John, H.; Bucher, C.; Engel, N.; Fischer, B.; Fehr, J.L. Preperitoneal robotic prostate adenomectomy. Urology 2009, 73, 811-815. [CrossRef]

14. Uffort, E.E.; Jensen, J.C. Robotic-assisted laparoscopic simple prostatectomy: An alternative minimal invasive approach for prostate adenoma. J. Robot Surg. 2010, 4, 7-10. [CrossRef]

15. Sutherland, D.E.; Perez, D.S.; Weeks, D.C. Robot-assisted simple prostatectomy for severe benign prostatic hyperplasia. J. Endourol. 2011, 25, 641-644. [CrossRef]

16. Coelho, R.F.; Chauhan, S.; Sivaraman, A.; Palmer, K.J.; Orvieto, M.A.; Rocco, B.; Coughlin, G.; Patel, V.R. Modified technique of robotic-assisted simple prostatectomy: Advantages of a vesico-urethral anastomosis. BJU Int. 2012, 109, 426-433. [CrossRef]

17. Vora, A.; Mittal, S.; Hwang, J.; Bandi, G. Robot-assisted simple prostatectomy: Multi-institutional outcomes for glands larger than 100 grams. J. Endourol. 2012, 26, 499-502. [CrossRef]

18. Fareed, K.; Zaytoun, O.M.; Autorino, R.; White, W.M.; Crouzet, S.; Yakoubi, R.; Haber, G.P.; White, M.A.; Kaouk, J.H. Robotic single port suprapubic transvesical enucleation of the prostate (R-STEP): Initial experience. BJU Int. 2012, 110, 732-737. [CrossRef] 
19. Matei, D.V.; Brescia, A.; Mazzoleni, F.; Spinelli, M.; Musi, G.; Melegari, S.; Galasso, G.; Detti, S.; de Cobelli, O. Robot-assisted simple prostatectomy (RASP): Does it make sense? BJU Int. 2012, 110, E972-E979. [CrossRef]

20. Clavijo, R.; Carmona, O.; De Andrade, R.; Garza, R.; Fernandez, G.; Sotelo, R. Robot-assisted intrafascial simple prostatectomy: Novel technique. J. Endourol. 2013, 27, 328-332. [CrossRef]

21. Leslie, S.; Abreu, A.L.; Chopra, S.; Ramos, P.; Park, D.; Berger, A.K.; Desai, M.M.; Gill, I.S.; Aron, M. Transvesical robotic simple prostatectomy: Initial clinical experience. Eur. Urol. 2014, 66, 321-329. [CrossRef]

22. Nestler, S.; Rubenwolf, P.; Neisius, A.; Thomas, C.; Roos, F.; Hampel, C.; Thüroff, J.W. Thüroff. Robot-Assisted Transvesical Enucleation of Benign Prostatic Hyperplasia: Lessons from a Single Surgeon's Learning Curve. Urol. Pract. 2014, 1, 40-44. [CrossRef]

23. Elsamra, S.E.; Gupta, N.; Ahmed, H.; Leavitt, D.; Kreshover, J.; Kavoussi, L.; Richstone, L. Robotic assisted laparoscopic simple suprapubic prostatectomy-The Smith Institute for Urology experience with an evolving technique. Asian J. Urol. 2014, 1, 55-59. [CrossRef]

24. Stolzenburg, J.U.; Kallidonis, P.; Qazi, H.; Ho Thi, P.; Dietel, A.; Liatsikos, E.N.; Do, M. Extraperitoneal approach for robotic-assisted simple prostatectomy. Urology 2014, 84, 1099-1105. [CrossRef]

25. Patel, M.N.; Hemal, A.K. Robot-assisted laparoscopic simple anatomic prostatectomy. Urol. Clin. N. Am. 2014, 41, 485-492. [CrossRef] [PubMed]

26. Nething, J.B.; Ricchiuti, D.J.; Irvine, R.; Drevna, D. Robotic simple prostatectomy: A consideration for large prostate adenomas. Arch. Ital. Urol. Androl. 2014, 86, 241-244. [CrossRef] [PubMed]

27. Pokorny, M.; Novara, G.; Geurts, N.; Dovey, Z.; De Groote, R.; Ploumidis, A.; Schatteman, P.; de Naeyer, G.; Mottrie, A. Robot-assisted simple prostatectomy for treatment of lower urinary tract symptoms secondary to benign prostatic enlargement: Surgical technique and outcomes in a high-volume robotic centre. Eur. Urol. 2015, 68, 451-457. [CrossRef] [PubMed]

28. Castillo, O.; Vidal-Mora, I.; Rodriguez-Carlin, A.; Silva, A.; Schatloff, O.; Borgna, V. Modified urethrovesical anastomosis during robot-assisted simple prostatectomy: Technique and results. Prostate Int. 2016, 4, 61-64. [CrossRef] [PubMed]

29. Cacciamani, G.; Medina, L.; Ashrafi, A.; Landsberger, H.; Winter, M.; Mekhail, P.; Desai, M.; Aron, M.; Berger, A. Transvesical robot-assisted simple prostatectomy with 360 degrees circumferential reconstruction: Step-by-step technique. BJU Int. 2018, 122, 344-348. [CrossRef]

30. Wang, P.; Xia, D.; Ye, S.; Kong, D.; Qin, J.; Jing, T.; Mao, Y.; Meng, H.; Wang, S. Robotic-assisted Urethra-sparing Simple Prostatectomy Via an Extraperitoneal Approach. Urology 2018, 119, 85-90. [CrossRef]

31. Chavali, J.S.; Garisto, J.; Bertolo, R.; Agudelo, J.; Kaouk, J.H. Surgical Hints for Robot-Assisted Transvesical Simple Prostatectomy. Urology 2018, 122, 185. [CrossRef]

32. Simone, G.; Misuraca, L.; Anceschi, U.; Minisola, F.; Ferriero, M.; Guaglianone, S.; Tuderti, G.; Gallucci, M. Urethra and Ejaculation Preserving Robot-assisted Simple Prostatectomy: Near-infrared Fluorescence Imaging-guided Madigan Technique. Eur. Urol. 2019, 75, 492-497. [CrossRef] [PubMed]

33. Johnson, B.; Sorokin, I.; Singla, N.; Roehrborn, C.; Gahan, J.C. Determining the Learning Curve for Robot-Assisted Simple Prostatectomy in Surgeons Familiar with Robotic Surgery. J. Endourol. 2018, 32, 865-870. [CrossRef] [PubMed]

34. Steinberg, R.L.; Passoni, N.; Garbens, A.; Johnson, B.A.; Gahan, J.C. Initial experience with extraperitoneal robotic-assisted simple prostatectomy using the da Vinci SP surgical system. J. Robot Surg. 2019. [CrossRef]

35. Dubey, D.; Hemal, A.K. Robotic-assisted simple prostatectomy with complete urethrovesical reconstruction. Indian J. Urol. 2012, 28, 231-232. [CrossRef]

36. Hoy, N.Y.; Van Zyl, S.; St Martin, B.A. Initial Canadian experience with robotic simple prostatectomy: Case series and literature review. Can. Urol. Assoc. J. 2015, 9, E626-E630. [CrossRef]

37. Autorino, R.; Zargar, H.; Mariano, M.B.; Sanchez-Salas, R.; Sotelo, R.J.; Chlosta, P.L.; Castillo, O.; Matei, D.V.; Celia, A.; Koc, G.; et al. Perioperative Outcomes of Robotic and Laparoscopic Simple Prostatectomy: A European-American Multi-institutional Analysis. Eur. Urol. 2015, 68, 86-94. [CrossRef]

38. Martin Garzon, O.D.; Azhar, R.A.; Brunacci, L.; Ramirez-Troche, N.E.; Medina Navarro, L.; Hernandez, L.C.; Nunez Bragayrac, L.; Sotelo Noguera, R.J. One-Year Outcome Comparison of Laparoscopic, Robotic, and Robotic Intrafascial Simple Prostatectomy for Benign Prostatic Hyperplasia. J. Endourol. 2016, 30, 312-318. [CrossRef] 
39. Pavan, N.; Zargar, H.; Sanchez-Salas, R.; Castillo, O.; Celia, A.; Gallo, G.; Sivaraman, A.; Cathelineau, X.; Autorino, R. Robot-assisted Versus Standard Laparoscopy for Simple Prostatectomy: Multicenter Comparative Outcomes. Urology 2016, 91, 104-110. [CrossRef]

40. Umari, P.; Fossati, N.; Gandaglia, G.; Pokorny, M.; De Groote, R.; Geurts, N.; Goossens, M.; Schatterman, P.; De Naeyer, G.; Mottrie, A. Robotic Assisted Simple Prostatectomy versus Holmium Laser Enucleation of the Prostate for Lower Urinary Tract Symptoms in Patients with Large Volume Prostate: A Comparative Analysis from a High Volume Center. J. Urol. 2017, 197, 1108-1114. [CrossRef]

41. Zhang, M.W.; El Tayeb, M.M.; Borofsky, M.S.; Dauw, C.A.; Wagner, K.R.; Lowry, P.S.; Bird, E.T.; Hudson, T.C.; Lingeman, J.E. Comparison of Perioperative Outcomes Between Holmium Laser Enucleation of the Prostate and Robot-Assisted Simple Prostatectomy. J. Endourol. 2017, 31, 847-850. [CrossRef]

42. Sorokin, I.; Sundaram, V.; Singla, N.; Walker, J.; Margulis, V.; Roehrborn, C.; Gahan, J.C. Robot-Assisted Versus Open Simple Prostatectomy for Benign Prostatic Hyperplasia in Large Glands: A Propensity Score-Matched Comparison of Perioperative and Short-Term Outcomes. J. Endourol. 2017, 31, 1164-1169. [CrossRef]

43. Mourmouris, P.; Keskin, S.M.; Skolarikos, A.; Argun, O.B.; Karagiannis, A.A.; Tufek, I.; Obek, C.; Riza Kural, A. A prospective comparative analysis of robot-assisted vs open simple prostatectomy for benign prostatic hyperplasia. BJU Int. 2019, 123, 313-317. [CrossRef]

44. Nestler, S.; Bach, T.; Herrmann, T.; Jutzi, S.; Roos, F.C.; Hampel, C.; Thuroff, J.W.; Thomas, C.; Neisius, A. Surgical treatment of large volume prostates: A matched pair analysis comparing the open, endoscopic (ThuVEP) and robotic approach. World J. Urol. 2019, 37, 1927-1931. [CrossRef]

45. Parsons, J.K.; Rangarajan, S.S.; Palazzi, K.; Chang, D. A National, Comparative Analysis of Perioperative Outcomes of Open and Minimally Invasive Simple Prostatectomy. J. Endourol. 2015, 29, 919-924. [CrossRef]

46. Vince, R.; Hampton, L.J.; Vartolomei, M.D.; Shariat, S.F.; Porpiglia, F.; Autorino, R. Robotic assisted simple prostatectomy: Recent advances. Curr. Opin. Urol. 2018, 28, 309-314. [CrossRef]

47. Dixon, A.R.; Lord, P.H.; Madigan, M.R. The Madigan prostatectomy. J. Urol. 1990, 144, 1401-1403. [CrossRef]

48. Lucca, I.; Shariat, S.F.; Hofbauer, S.L.; Klatte, T. Outcomes of minimally invasive simple prostatectomy for benign prostatic hyperplasia: A systematic review and meta-analysis. World J. Urol. 2015, 33, 563-570. [CrossRef]

49. Li, J.; Cao, D.; Peng, L.; Ren, Z.; Gou, H.; Li, Y.; Wei, Q. Comparison Between Minimally Invasive Simple Prostatectomy and Open Simple Prostatectomy for Large Prostates: A Systematic Review and Meta-Analysis of Comparative Trials. J. Endourol. 2019, 33, 767-776. [CrossRef]

50. Brunckhorst, O.; Ahmed, K.; Nehikhare, O.; Marra, G.; Challacombe, B.; Popert, R. Evaluation of the Learning Curve for Holmium Laser Enucleation of the Prostate Using Multiple Outcome Measures. Urology 2015, 86, 824-829. [CrossRef]

51. Leonardo, C.; Lombardo, R.; Cindolo, L.; Antonelli, A.; Greco, F.; Porreca, A.; Veneziano, D.; Pastore, A.; Dalpiaz, O.; Ceruti, C.; et al. What is the standard surgical approach to large volume BPE? Systematic review of existing randomized clinical trials. Minerva Urol. Nefrol. 2020, 72, 22-29. [CrossRef]

52. Ferretti, M.; Phillips, J. Prostatectomy for benign prostate disease: Open, laparoscopic and robotic techniques. Can. J. Urol. 2015, 22 (Suppl. 1), 60-66.

53. Ou, R.; You, M.; Tang, P.; Chen, H.; Deng, X.; Xie, K. A randomized trial of transvesical prostatectomy versus transurethral resection of the prostate for prostate greater than $80 \mathrm{~mL}$. Urology 2010, 76, 958-961. [CrossRef] [PubMed]

54. Zhu, G.; Xie, C.; Wang, X.; Tang, X. Bipolar plasmakinetic transurethral resection of prostate in 132 consecutive patients with large gland: Three-year follow-up results. Urology 2012, 79, 397-402. [CrossRef] [PubMed]

55. Autorino, R.; Zargar, H.; Kaouk, J.H. Robotic-assisted laparoscopic surgery: Recent advances in urology. Fertil. Steril. 2014, 102, 939-949. [CrossRef]

56. Bach, T.; Wolbling, F.; Gross, A.J.; Netsch, C.; Tauber, S.; Pottek, T.; Wulfing, C.; Brunken, C. Prospective assessment of perioperative course in 2648 patients after surgical treatment of benign prostatic obstruction. World J. Urol. 2017, 35, 285-292. [CrossRef]

57. Jones, P.; Alzweri, L.; Rai, B.P.; Somani, B.K.; Bates, C.; Aboumarzouk, O.M. Holmium laser enucleation versus simple prostatectomy for treating large prostates: Results of a systematic review and meta-analysis. Arab. J. Urol. 2016, 14, 50-58. [CrossRef]

58. De Carvalho, P.A.; Coelho, R.F. Surgical Treatment: Robotic Simple Prostatectomy. Big Prostate 2018. [CrossRef] 
59. Pariser, J.J.; Pearce, S.M.; Patel, S.G.; Bales, G.T. National Trends of Simple Prostatectomy for Benign Prostatic Hyperplasia With an Analysis of Risk Factors for Adverse Perioperative Outcomes. Urology 2015, 86, 721-725. [CrossRef]

60. Salonia, A.; Suardi, N.; Naspro, R.; Mazzoccoli, B.; Zanni, G.; Gallina, A.; Bua, L.; Scattoni, V.; Rigatti, P.; Montorsi, F. Holmium laser enucleation versus open prostatectomy for benign prostatic hyperplasia: An inpatient cost analysis. Urology 2006, 68, 302-306. [CrossRef]

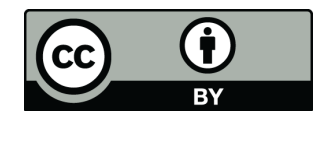

(C) 2020 by the authors. Licensee MDPI, Basel, Switzerland. This article is an open access article distributed under the terms and conditions of the Creative Commons Attribution (CC BY) license (http://creativecommons.org/licenses/by/4.0/). 